\title{
Singularity theorems for the Reissner-Nordström spacetime and topology change in regular black holes
}

\author{
Pedro Bargueño ${ }^{*}$ \\ Departamento de Física Aplicada, Universidad de Alicante, \\ Campus de San Vicente del Raspeig, E-03690 Alicante, Spain
}

(Received 26 March 2021; accepted 30 June 2021; published 26 July 2021)

\begin{abstract}
In this work we show several singularity theorems which can be applied to the Reissner-Nordström spacetime. To date, only two results [Phys. Rev. D 50, 3692 (1994); Lett. Math. Phys. 110, 2383 (2020)] have predicted null incompleteness in this case. In addition, we show that regular black holes in the Bardeen category have noncompact slices without the standard assumption regarding the knowledge of regions at infinity and, therefore, that they exhibit topology changes.
\end{abstract}

DOI: 10.1103/PhysRevD.104.024063

\section{INTRODUCTION}

Although Penrose's theorem [1] is one of the cornerstones of rigorous results in gravitational physics, some of its assumptions have been considered too strong for a realistic universe. In particular, global hyperbolicity seems to be at odds with the unpredictability inherent to quantum theories. Nevertheless, Borde noticed [2,3] that what is essential for the validity of the theorem is not global hyperbolicity but some kind of topological condition to prevent light rays from wrapping around the Universe in the crucial region where trapped surfaces occur. Technically, the singularity predicted by Penrose is avoided because there is a compact achronal slice. Very recently, Minguzzi [4] showed that the causality conditions in Penrose's theorem can be almost completely removed, thus enabling one to obtain a singularity theorem which is, in principle, compatible with black hole evaporation. The essential point lies in substituting global hyperbolicity by past reflectivity and the noncompact Cauchy hypersurface condition for a spatially open spacetime [4].

In addition to Penrose's theorem, the multipurpose Hawking and Penrose theorem [5] is also considered a classical result. Although this theorem was originally conceived to embrace generic situations, the adoption of the "generic condition" had the effect of excluding special situations, some of them being very relevant, as for instance the Reissner-Nordström solution, where this condition is violated along radial null geodesics (the interested reader can find more details on this specific condition in Senovilla's review [6]). Penrose's theorem does not apply in this case either due to the global hyperbolicity condition. Interestingly, to our knowledge, only two results predict null incompleteness in the

*pedro.bargueno@ua.es
Reissner-Nordström case: a theorem by Borde [2] based basically on the existence of localized light cones in a stably causal and causally simple spacetime possessing a trapped surface, and Miguzzi's result [4], as previously commented.

It is our purpose here to show several singularity theorems which we have designed to be valid for the ReissnerNordström case. This is of interest because, while some standard assumptions are retained, a scarcely known result (the Malament-Hogarth property) is included to prove one of the desired results, with the other being based on standard techniques. As a consequence, here we show that regular black holes (in the sense of Ref. [3]) with either the Malament-Hogarth property, past reflectivity or being nonglobally hyperbolic exhibit topology changes without the standard assumption regarding the knowledge of regions at infinity. This result reinforces some previous corollaries established in Ref. [7] to clarify whether topology change in regular black holes needs some asymptotically flat region in order to occur.

This work is organized as follows. Section II introduces some definitions and preliminary results of global differential geometry and topology in Lorentzian manifolds which are needed to prove the desired theorems. In particular, the definitions of past reflectivity, unavoidability, and the Malament-Hogarth property, which, although scarcely known, we think are of interest to the relativistic community, are briefly reviewed here. In essence, the purpose of Sec. II is to provide the reader a self-content introduction in order to avoid looking for preliminary results across the vast literature. Then the main results are stated in Sec. III, where sketches of some of the proofs are provided in order not to lose the continuity of the ideas. In particular, this section contains Theorems 2 and 3 and Corollaries 1 and 2, which show, respectively, that the Reissner-Nordström spacetime is singular and that 
topology change does not depend on the asymptotic structure of the corresponding regular black hole, which is usually required in order to conclude that topology change appears. This is an important result which is applicable to most of the regular black holes considered in the literature, as we will show throughout the manuscript. Section IV deals with results equivalent to those shown in Sec. III but introducing a weaker hypothesis (Minguzzi's past reflectivity) of relevance in the context of evaporating black holes. Some comments regarding the class of metrics considered in the standard singularity (and regularity) theorems are made in Sec. V. Finally, we conclude with a brief summary of the main results and some comments and suggestions for future work in Sec. VI.

\section{PRELIMINARY DEFINITIONS}

Let us go into detail by introducing some of the relevant definitions and notation [8]. In this work, a spacetime is a pair $(\mathcal{M}, g)$ where $\mathcal{M}$ is a connected four-dimensional Hausdorff $C^{\infty}$ manifold and $g$ is a Lorentz metric on $\mathcal{M}$ (for brevity we will refer to $\mathcal{M}$ as a spacetime without explicit reference to the Lorentz metric, although some comments regarding its regularity class will be made at the end of the manuscript). The chronological future of $p \in \mathcal{M}, I^{+}(p)$ is the set of all $q \in \mathcal{M}$ such that there is a smooth future-directed nondegenerate timelike curve from $p$ to $q$. In case the preceding curve is causal (allowing the possibility of being degenerate) we define the causal future of $p, J^{+}(p)$. Given two sets, $U$ and $V$, the chronological (causal) future of $U$ relative to $V$ is defined as the set of all points of $V$ which can be reached from $U$ by a future-directed timelike (causal) curve in $V$. A subset $S \subset \mathcal{M}$ of an arbitrary spacetime $\mathcal{M}$ is said to be achronal if there is not a pair $p, q \in S$ such that it can be connected by timelike curves. Let $S$ be an achronal subset of $\mathcal{M}$. Then $p \in \bar{S}$ is an edge point of $S$ provided that every neighborhood $U$ of $p$ contains a timelike curve $\gamma$ from $I^{-}(p, U)$ to $I^{+}(p, U)$ that does not meet $S$. A slice $\Gamma$ is an edgeless, achronal hypersurface. Let $\mathcal{S}$ be a spacelike three-manifold. If every inextendible nonspacelike curve in $\mathcal{M}$ intersects $\mathcal{S}$, then $\mathcal{S}$ is said to be a Cauchy surface. A partial Cauchy surface is a closed achronal set $S$ without an edge (thus, a spacelike hypersurface). $\mathcal{M}$ is said to be globally hyperbolic if it admits a global Cauchy surface. In this case, from Geroch's splitting theorem [9], $\mathcal{M}$ is homeomorphic to $\mathbb{R} \times \mathcal{S}$ (the extensions to the diffeomorphism were developed in Ref. [10]). The future domain of dependence of a set $A$ is given by $D^{+}(A)$, which is the set of $p \in \mathcal{M}$ such that every past-directed endless causal curve from $p$ meets $A$. The total domain of dependence is given by $D(A) \equiv D^{+}(A) \cup D^{-}(A)$. The future Cauchy horizon of $A$ is $H^{+}(A)=\overline{D^{+}(A)}-I^{-}\left[D^{+}(A)\right]$. Equivalently, the total Cauchy horizon of $A$ is $H(A) \equiv H^{+}(A) \cup H^{-}(A)$. A spacetime $\mathcal{M}$ is said to be future causally simple if
$E^{+}(X)=\dot{I}^{+}(X)$, where $\dot{I}^{+}(X)$ is the boundary of $I^{+}(X)$ and $X$ is some compact achronal subset of $\mathcal{M} . E^{+}(X)$ is the future horismo of $X$, which is defined by $E^{+}(X)=J^{+}(X)-I^{+}(X)$. We say that $\mathcal{M}$ satisfies the strong causality condition at $p \in \mathcal{M}$ if there are arbitrarily small neighborhoods of $p$ which no future-directed causal curve intersects in a disconnected set. A chronology violating set $\mathcal{C}$ consists of all points $p \in \mathcal{M}$ through which a closed timelike curve passes. Note that, on a subset $S$, the condition " $S$ is achronal" is stronger than $S \cup \mathcal{C}=\varnothing$. Regarding not the causal but the transverse ladder [11], which imposes some relational or topological conditions on the causal relations, we will need the following definition. $\mathcal{M}$ is said to be past reflecting if, for every $p, q \in \mathcal{M}$, $I^{+}(q) \subset I^{+}(q) \Rightarrow I^{-}(p) \subset I^{-}(q)$ (for equivalent definitions, see Definition 4.6 of [11]). A trapped surface is a two-surface in which both outgoing and ingoing null geodesics perpendicular to this surface are convergent, i.e., these null geodesics have negative divergence on this surface (although the causal orientation of the mean curvature vector provides a better and more powerful characterization of the trapped surfaces [12], for our purposes we will adhere to the original definition). For an eventually future-trapped surface the only requirement is that the divergences be negative somewhere in the future of the surface along each geodesic [3]. To capture the idea of a trapped set that swallows the entire Universe [2,3], a compact nonempty set $S$ that does not intersect $C$ is said to have an unavoidable or swallowing future horismos if there is an open neighborhood $U$ of $E^{+}(S)$ such that $I_{U}^{-}\left(E^{+}(S)\right) \subset \operatorname{Int} D^{-}\left(E^{+}(S)\right)$ [4]. The Ricci tensor obeys the null convergence condition if $R_{\mu \nu} n^{\mu} n^{\nu} \geq 0$ for all null vectors $n^{\mu}$. We say that a spacetime $\mathcal{M}$ is nonspacelike geodesically incomplete if $\mathcal{M}$ has a timelike or null geodesic which cannot be defined for all values of an affine parameter. These spacetimes are said to be singular.

Finally, let us introduce the following.

Definition [13].-A spacetime $\mathcal{M}$ has the MalamentHogarth (MH) property when there is a future endless timelike curve $\lambda \in \mathcal{M}$ with a past end point and a point $q \in \mathcal{M}$ such that $\int_{\lambda} d s=\infty$ and $\lambda \in J^{-}(q)$, This definition basically states that a spacetime has the MH property if it admits a point in the future of a curve with a past end point and infinite length.

\section{MAIN RESULTS}

Some properties and examples of spacetimes with the $\mathrm{MH}$ property which are relevant for our purposes are stated here without proof (as proof can be found in the corresponding references).

Proposition 1 [13].-A spacetime with the MH property is not globally hyperbolic.

Additionally, one of the key results states that no spacetime with the MH property can be spatially closed at any time in its history. 
Proposition 2 [14].-A spacetime with the MH property admits a noncompact slice [specifically, $\dot{I}^{-}(\lambda)$ is the aforementioned slice].

Physically relevant examples of spacetimes with the $\mathrm{MH}$ property were pointed out in the original references [13,14], where they were shown to play an important role to implement "supertasks" (the interested reader can consult a brief and physically motivated introduction to these issues in [15]). They include anti-de Sitter and Reissner-Nordström spacetimes, as the reader can easily check with an explicit calculation in the first case and by using the corresponding Carter-Penrose diagram in the second one [13]. In the latter case, the idea is to draw a future-inextendable timelike curve, $\lambda$, contained in an asymptotically flat region extending from a given point to the timelike infinity of that region. Then $\lambda$ lies to the past of $q$, which is a point on the Cauchy horizon. Using the same trick, Etesi and Németi showed [16] that both Kerr and Kerr-Newman spacetimes have the $\mathrm{MH}$ property.

Let us recall that the previously mentioned results of Borde [2,3], which have had considerable impact with respect to the so-called regular black holes, i.e., geodesically complete spacetimes possessing trapped surfaces and inflationary cores, which essentially refers to black holes possessing a de Sitter-like structure substituting the singularity (see, for example, Refs. [7,17] and references therein), are based on the following "reversed" Penrose's theorem.

Theorem 1 (Borde) [3].--Let $\mathcal{M}$ be a spacetime such that the following hold:

(1) It contains an eventually future-trapped surface $\mathcal{T}$.

(2) The null convergence condition is satisfied.

(3) It will be null-geodesically complete in the future.

(4) It is future causally simple with $E^{+}(X) \neq \varnothing$ for all achronal, compact $X \subset \mathcal{M}$.

Then there is a compact slice to the causal future of $\mathcal{T}$.

See [3] for a proof of this.

Here we note that a plethora of regular black hole models showing the applicability of Theorem 1 have appeared in the literature [7,18-22], most of them sharing an $S^{3}$ compact achronal slice as a common feature.

One of our main results now follows from Proposition 2 and Theorem 1.

Theorem 2.-Let $\mathcal{M}$ be a spacetime such that the following hold:

(1) It contains an eventually future-trapped surface.

(2) The null convergence condition is satisfied.

(3) It has the MH property.

(4) It is future causally simple.

(5) It does not admit both open and compact slices.

Then $\mathcal{M}$ will be null-geodesically incomplete in the future.

At this point, some comments are in order: (i) The Reissner-Nordström solution turns to be null-geodesically incomplete under the hypothesis of the previous theorem, and (ii) as a side bonus we can enunciate the following.

Corollary 1.-Topology change is mandatory if a regular black hole (in the sense of Theorem 1) has the MH property.

Specifically, as stated by Borde [3]: "The theorem [Theorem 1], as stated, does not directly make a statement about topology change. A black hole spacetime, however, usually contains a region at infinity and may therefore be expected to start with a noncompact slice $S$, the black hole, and, hence, at least one eventually future-trapped surface in the future. The theorem shows, under very general conditions, that if the black hole is to be regular, the spacetime must develop a compact slice to the future of S: i.e., the topology must change from open to closed."

In this sense, Corollary 1 shows that topology change does not depend on the asymptotic structure of the corresponding black hole, which is usually required in order to conclude that topology change appears, but the MH property is a sufficient condition (given the rest of the hypothesis). As this property is satisfied by all regular black holes of the Bardeen type, which are strongly based on the Reissner-Nordström solution once the region near the singularity has been substituted for a de Sitter core, we can conclude that topology change does occur for this kind of black holes.

Interestingly, an alternative way of showing null incompleteness which does not rely on the MH property can be done along the following lines.

Let $\mathcal{M}$ be a spacetime with a Cauchy horizon $H$. Now consider the following results.

Lemma 1 (adapted from Lemma 2.7 of Ref. [6] and Lemma 8.15 of Ref. [23]). - Let $X$ be an achronal and closed subset of $\mathcal{M}$. Then strong causality holds on $\operatorname{int} D(X)$, and therefore $H^{+}\left[E^{+}(X)\right]$ is noncompact or empty.

Lemma 2 [see [11], Definition 3.14, Proposition 3.15, Proposition 3.22, Theorem 3.24, and Eq. (3.6)].- - Let $X$ be a closed achronal subset of $\mathcal{M}$. Then $H^{+}(X)$ is achronal and closed and edge $(X)=\operatorname{edge}\left(H^{+}(X)\right)$.

Therefore, from Theorem 1 and Lemmas 1 and 2, the following result holds.

Theorem 3.-Let $\mathcal{M}$ be a nonglobally hyperbolic spacetime such that the following hold.

(1) It contains an eventually future-trapped surface.

(2) The null convergence condition is satisfied.

(3) It is future causally simple.

(4) $H^{+} \overline{\left[E^{+}(X)\right]} \neq \varnothing$ with $X \subset \mathcal{M}$ closed and achronal.

(5) It does not admit both open and compact slices.

Then $\mathcal{M}$ will be null-geodesically incomplete in the future.

Proof of Theorems 2 and 3.-Theorem 2 is a combination of Theorem 1 and the hypothesis of the MH property along with a topological condition (the spacetime does not admit open and compact slices). Theorem 3 is a consequence of Lemmas 1 and 2, a topological condition (the spacetime does 
not admit open and compact slices) and Theorem 1. In both cases, one can construct a noncompact slice (either as a consequence of the $\mathrm{MH}$ property or by taking the future horizon of the closure of the future horismos of an achronal and closed set). Moreover, Theorem 1 gives conditions for a spacetime to have a compact slice. Therefore, the only hypothesis in Theorem 1 that is not included in Theorems 2 and 3 is null-geodesically completeness in the future. As it is required for the spacetime not to admit open and compact slices, the spacetime must be null-geodesically incomplete in the future.

Note that this result can be applied to the ReissnerNordström solution, too. Additionally, in the same line of Corollary 1 we can state the following.

Corollary 2.-Topology change is mandatory if a regular black hole (in the sense of Theorem 1) is not globally hyperbolic (note that this result is valid for Bardeen-like regular black holes and also for those not lying in this category such as, for example, regular black holes with Nariai or Bertotti-Robinson cores [24]).

As a side comment, we mention that it can be seen that the $\mathrm{MH}$ property for a Bardeen-like spacetime is provided by any point $p \in H^{+}\left(E^{+}(S)\right)$, where $S$ is a trapped surface and $\lambda$ is a future-directed timelike curve from $S$ (region II) to the timelike infinity of region III (see Fig. 12 of [6]).

Finally, we note that it would be interesting to extend our results to the case of the Kerr spacetime, which fulfills all the hypotheses of Theorems 2 and 3 with the exception of causal simplicity. This could be done, in principle, by restoring it while considering only the exterior $(r \geq 0)$ region. Similar ideas were considered by Minguzzi so that Theorem 6 of Ref. [4] could be applied to the KerrNewman case.

\section{SOME COMMENTS REGARDING MINGUZZI'S RESULTS}

It is interesting to note that Minguzzi (Theorem 7 in [4]) provides a result similar to Theorem 1 with a weaker hypothesis [the spacetime must only be past reflecting (see Definition 4.6 of [11]) and not future causally simple]. Therefore, this theorem can also be used to formulate some results similar to that obtained in Theorem 2. Let us start by stating Minguzzi's result.

Theorem 4 (Minguzzi) [4].- - Let $\mathcal{M}$ be a past reflecting spacetime which satisfies the null convergence condition. Suppose that it admits a future-trapped surface $S$ such that $S \cap \mathcal{C}=\varnothing$. Then it is either future null geodesically incomplete or the horismos $E^{+}(S)$ is compact, unavoidable, and $E^{+}(S)=\dot{I}^{+}(S)$.

Therefore, as causal simplicity can be safely substituted for past reflectivity in Theorem 2, we can state the following theorem.

Theorem 2(b).-Let $\mathcal{M}$ be a spacetime such that the following hold:
(1) It contains a future-trapped surface $S$ such that $S \cap \mathcal{C}=\varnothing$.

(2) The null convergence condition is satisfied.

(3) It has the MH property.

(4) It is past reflecting.

(5) It fails to admit both open and compact slices.

Then $\mathcal{M}$ is null-geodesically incomplete in the future.

This result also predicts the singularity in the ReissnerNordström solution and, as in the previous case, to the Kerr-Newman solution if the $r<0$ region is removed [4]. In addition, although Minguzzi's theorem can also be reversed in different ways by negating either causal simplicity or unavoidability, we do not explore these possibilities here.

Finally, we would like to note that we have not been able to either find an example where Theorem 2 works but Theorem 2(b) does not or find a result showing to which extent the two results are equivalent. The same applies to Theorem 2(b) and Minguzzi's singularity theorem (Theorem 6 in [4]), which is stated as follows.

Theorem 5 (Minguzzi) [4].- - Let $\mathcal{M}$ be a past reflecting spacetime which is open and satisfies the null convergence condition. Suppose that it admits a future-trapped surface $S$ such that $S \cap \mathcal{C}=\varnothing$. It is then future null geodesically incomplete.

Interestingly, by reversing Theorem 5 we can obtain the following corollary.

Corollary 3.- Let $\mathcal{M}$ be a future null geodesically complete and past reflecting spacetime which satisfies the null convergence condition. Suppose that it admits a future-trapped surface $S$ such that $S \cap \mathcal{C}=\varnothing$. It then admits a compact slice.

Therefore, the causal simplicity condition can be dropped in order to show that topology change is a consequence of considering regular black holes in the sense of Theorem 5 .

\section{ON THE REGULARITY OF THE METRIC}

In general, Penrose [1], Hawking [25], and Hawking and Penrose's singularity theorems [5] hold for $C^{2}$ Lorentzian metrics. However, these results have recently been extended for metrics that are $C^{1,1}$ (metrics that are differentiable, with all derivatives locally Lipschitz) [26-28], which is of both mathematical and physical significance. In a case in which the regularity of the metric drops to $C^{1,1}$, the classical theorems would predict that the curvature would become discontinuous rather than unbounded, which corresponds to a finite jump in the matter variables, and therefore such a situation would hardly be regarded as singular. This is one of the reasons behind the importance of the aforementioned generalizations.

As shown in the manuscript, the results obtained here rely on Borde's [3] and Minguzzi's [4] theorems. In the first case, although not explicitly stated in Borde's original work, 
the metric is assumed to be $C^{2}$ since Borde's theorem is strongly based on the original Penrose one [1], which originally assumed $C^{2}$ regularity. In this sense, Theorem 2 assumes a $C^{2}$ Lorentzian metric. In the second case, Minguzzi extends Penrose's original theorem by substituting global hyperbolicity using past reflectivity, and the noncompact Cauchy hypersurface condition using a spatially open spacetime. Therefore, the assumption of considering $C^{2}$ metrics is also hidden in Ref. [4], and therefore this is also the regularity class employed in Theorem 2(b). Although it might be reasonable to conjecture that a $C^{1,1}$ version of Borde and Minguzzi's theorems and the consequences obtained here could be stated based on Ref. [27], we leave the proof for the interested reader.

\section{SUMMARY, FINAL COMMENTS, AND SUGGESTIONS FOR FUTURE WORK}

Here we will enumerate the novel and significant physics issues that have been addressed in the manuscript in order to make the paper more accessible to readers without having to go into technical details.

(1) We have proved several singularity theorems designed to detect future null incompleteness for the ReissnerNordström spacetime, which was not captured either by Penrose's or Hawking and Penrose's theorem.

(2) These results have been obtained by extending previous works by Borde and Minguzzi. In particular, we have employed for the first time the Malament-Hogarth property as a hypothesis in a singularity theorem.

(3) The inclusion of Miguzzi's past reflectivity has allowed us to extend our results to the case of evaporating black holes, which are not causally simple (one of the hypotheses usually invoked to obtain regular black holes).

(4) We have shown that regular black holes in the Bardeen category have noncompact slices without the standard assumption regarding the knowledge of regions at infinity.

(5) As a consequence, we have shown that regular black holes exhibit topology changes irrespective or their asymptotic structure.

Finally, here we will delineate some open questions related to this work:

(a) Extend the results to the Kerr and Kerr-Newman cases.

(b) Find an example where Theorem 2 works but Theorem 2(b) does not or display a result showing to what extent the two results are equivalent.

(c) Prove a version of Borde and Miguzzi's theorems assuming $C^{1,1}$ regularity for the metric.

(d) How is topology change in regular black holes reflected in physical quantities? (Hint: construct an appropriate Morse function from scalar quantities of physical relevance describing the regular black hole in order to track topology change.)

\section{ACKNOWLEDGMENTS}

P. B. acknowledges J. M. M. Senovilla for the useful correspondence, and the anonymous referee for valuable comments which have served to improve the presentation of this work. P. B. is funded by Beatriz Galindo Contract No. BEAGAL 18/00207 (Spain). P. B. acknowledges Miguel Angel Botella for having introduced him the world of spacetime warps and wripples.
[1] R. Penrose, Phys. Rev. Lett. 14, 57 (1965).

[2] A. Borde, Phys. Rev. D 50, 3692 (1994).

[3] A. Borde, Phys. Rev. D 55, 7615 (1997).

[4] E. Minguzzi, Lett. Math. Phys. 110, 2383 (2020).

[5] S. W. Hawking and R. Penrose, Proc. R. Soc. A 314, 529 (1970).

[6] J. M. M. Senovilla, Gen. Relativ. Gravit. 30, 701 (1998).

[7] P. Bargueno, Phys. Rev. D 102, 104028 (2020).

[8] S. W. Hawking and G. F. R. Ellis, The Large Scale Structure of Space-Time (Cambridge University Press, Cambridge, England, 1973).

[9] R. Geroch, J. Math. Phys. (N.Y.) 11, 437 (1970).

[10] A. N. Bernal and M. Sánchez, Commun. Math. Phys. 243, 461 (2003).

[11] E. Minguzzi, Living Rev. Relativity 22, 3 (2019).

[12] J. M. M. Senovilla, Classical Quantum Gravity 19, L113 (2002).
[13] M. L. Hogarth, Found. Phys. Lett. 5, 173 (1992).

[14] M. L. Hogarth, Ph. thesis, Sidney Sussex College, 1996.

[15] C. Berthiere, G. Gibbons, and S. N. Solodukhin, Phys. Rev. D 92, 064036 (2015).

[16] G. Etesi and I. Németi, Int. J. Theor. Phys. 41, 341 (2002).

[17] R. Carballo-Rubio, F. Di Filippo, S. Liberati, and M. Visser, Phys. Rev. D 101, 084047 (2020).

[18] I. Dymnikova, Gen. Relativ. Gravit. 24, 235 (1992).

[19] I. Dymnikova, Classical Quantum Gravity 19, 725 (2002).

[20] S. A. Hayward, Phys. Rev. Lett. 96, 031103 (2006).

[21] V. P. Frolov, Phys. Rev. D 94, 104056 (2016).

[22] V. P. Frolov and A. Zelnikov, Phys. Rev. D 95, 124028 (2017).

[23] J. K. Beem, P. E. Ehrlich, and K. L. Easley, Global Lorentzian Geometry, Monographs and Textbooks in Pure and Applied Mathematics Vol. 202 (Marcel Dekker, New York, 1996). 
[24] G. Melgarejo, E. Contreras, and P. Bargueno, Phys. Dark. Universe 30, 100709 (2020).

[25] S. W. Hawking, Proc. R. Soc. A 300, 187 (1967).

[26] M. Kunzinger, R. Steinbauer, M. Stojkovi, and J. A. Vickers, Classical Quantum Gravity 32, 075012 (2015).
[27] M. Kunzinger, R. Steinbauer, and J. A. Vickers, Classical Quantum Gravity 32, 055010 (2015).

[28] M. Graf, J. D. E. Grant, M. Kunzinger, and R. Steinbauer, Commun. Math. Phys. 360, 1009 (2018). 